\title{
Multisensory perception and attention in school-age children
}

Ayla Barutchu ${ }^{1}$, Sarah Toohey ${ }^{2}$, Mohit N. Shivdasani ${ }^{6,3}$, Joanne M. Fifer ${ }^{2}$, Sheila G. Crewther $^{2}$, David B. Grayden ${ }^{4,3}$ \& Antonio G. Paolini ${ }^{2,5}$

${ }^{1}$ Balliol College, University of Oxford, UK, OX1 3BJ

${ }^{2}$ School of Psychology and Public Health, La Trobe University, VIC 3086, Australia

${ }^{3}$ Bionics Institute, East Melbourne, VIC 3002, Australia

${ }^{4}$ Department of Biomedical Engineering, The University of Melbourne, VIC 3010, Australia

${ }^{5}$ ISN Psychology, Institute for Social Neuroscience, VIC 3084, Australia

${ }^{6}$ Graduate School of Biomedical Engineering, The University of New South Wales, NSW 2033, Australia

The authors have no conflict of interest to declare.

\section{Corresponding Author:}

Ayla Barutchu, PhD

Balliol College

University of Oxford,

Oxford, OX1 3UD

Email: ayla.barutchu@balliol.ox.ac.uk 
Research highlights:

- Multisensory processes in children are significantly related to attention abilities.

- The McGurk Effect, where vision alters auditory perception, is more pronounced in children with better visual-spatial and audiovisual dual attention abilities.

- The Stream-Bounce Effect, where sound alters visual perception, is more pronounced in children with better auditory sustained attention.

- In school-age children, the relationship between attention type and multisensory processing is flexible and task-dependent. 


\begin{abstract}
Although it is well known that attention can modulate multisensory processes in adults and infants, this relationship has not been investigated in school-age children. Attention abilities of 53 children (ages 7-13) were assessed using three subscales of the Test of Everyday Attention for Children (TEA-Ch): visual spatial attention (symbol search -SS), auditory sustained attention (Score), and auditory-visual dual task (SSDT, where the SS and the Score tasks are performed simultaneously). Multisensory processes were assessed using the McGurk Effect (a verbal illusion where speech perception is altered by vision) and the Stream-Bounce (SB) Effect (a non-verbal illusion where visual perception is altered by sound). The likelihood of perceiving both multisensory illusions tended to increase with age. The McGurk Effect was significantly more pronounced in children who scored high on the audiovisual dual attention indices (SSDT). In contrast, the SB Effect was more pronounced in children with higher sustained auditory attention abilities as assessed by the Score index. These relationships between attention and the multisensory illusory percepts could not be explained solely by age or children’s intellectual abilities. This study suggests that the interplay between attention and multisensory processing depends on both the nature of the multisensory task and the type of attention needed to effectively merge information across the senses.
\end{abstract}

Word count: 215 (250 max)

Keywords: attention, multisensory processing, vision, audition, illusion, children. 
Like most species, humans live, learn, and develop in multisensory environments. Multisensory stimuli in close spatial and temporal proximity not only have enhancing effects on information processing throughout the life span (e.g., Bahrick \& Lickliter, 2000; Barutchu, Crewther, \& Crewther, 2009; Barutchu et al., 2011), but can also lead to illusory phenomena (e.g., McGurk \& MacDonald, 1976; Sekuler, Sekuler, \& Lau, 1997), providing compelling evidence that information across the sensory systems is merged into a unified percept. Multisensory illusions can be speech-based, such as the McGurk Effect (the lip movements of /ga/ coupled with the sound /ba/ leads to the perception of /da/) (McGurk \& MacDonald, 1976), or non-speech-based, such as the Stream-Bounce (SB) Effect (an ambiguous display of two objects moving toward each other are more likely to be perceived as bouncing or colliding objects than streaming or passing objects with the presentation of a brief sound when the objects touch) (Sekuler et al., 1997). The verbally based McGurk Effect results when auditory speech perception is altered by vision while the SB Effect occurs when visual perception is altered by sound.

Past research suggests that multisensory processes that lead to behavioral enhancements or illusory phenomena develop slowly throughout late childhood and adolescence (e.g., Barutchu et al., 2009; Brandwein et al., 2011; Bremner, Lewkowicz, \& Spence, 2012; Downing, Barutchu, \& Crewther, 2014; Gori, Del Viva, Sandini, \& Burr, 2008; McGurk \& MacDonald, 1976; Nardini, Bales, \& Mareschal, 2016; Nardini, Bedford, \& Mareschal, 2010; Ross et al., 2011). Furthermore, although audiovisual processes in adults are optimal for stimuli in close temporal proximity, studies have suggested that the temporal binding window may be wider in children than adults; it has been shown to have a protracted developmental course throughout childhood and into adolescence (Chen, Shore, Lewis, \& Maurer, 2016; Hillock-Dunn 
\& Wallace, 2012). The developmental trajectories of multisensory processes may also be dissociable depending on the task and the signal type (e.g., speech vs. non-speech) (Tremblay et al., 2007). Indeed, there is a limited understanding of how multisensory information is merged and related to the development of other cognitive processes, such as attention.

Attention refers to the ability to direct and maintain focus on an object, task, or event and inhibit distractions (Goldberg \& Wurtz, 1972; James, 1890). In adults, although attention mechanisms have been shown to be interlinked across the sensory systems, they still maintain some level of independence (e.g., Driver \& Spence, 1998; Spence \& Driver, 1996). Furthermore, in adults, multisensory processes not only have an enhancing effect on orienting attention (e.g., Hillyard, Stormer, Feng, Martinez, \& McDonald, 2016; Spence \& Driver, 1996), but attention has been shown to be a requisite for the integration of information across the senses (e.g., Alsius, Navarra, Campbell, \& Soto-Faraco, 2005; Andersen, Tiippana, Laarni, Kojo, \& Sams, 2009; Buchan \& Munhall, 2011; Talsma, 2015; Talsma, Senkowski, Soto-Faraco, \& Woldorff, 2010). The specific role of attention in multisensory processing, however, may differ with age and across multisensory processes. For example, in adults when attention is taxed and divided across dual tasks the McGurk Effect depletes (Alsius et al., 2005), suggesting that attention plays a binding role in audiovisual speech integration. Indeed the temporal congruence of audiovisual speech information and spatial attention have been shown to interact and influence, not only the strength of the McGurk Effect (Andersen et al., 2009; Buchan \& Munhall, 2011), but also the underlying complex neural network (Fairhall \& Macaluso, 2009). In contrast, the perception of streaming objects in the SB Effect is dependent on visual attention, and distractors that capture attention; The SB Effect requires a distractor like a transient 
sound at the point of collision, to bias perception to bouncing objects (Shimojo, Watanabe, \& Scheier, 2001). Thus, the perception of bouncing objects is dependent on audition capturing and biasing attention away from vision to fuse the audiovisual inputs into the perception of bouncing objects.

Similarly, it is well accepted that from the earliest post-natal stages, multisensory processes play an important role in guiding, orienting, and manipulating attention and learning (e.g., Bahrick \& Lickliter, 2000; Foreman \& Fielder, 1989; Muir \& Field, 1979). However, the interplay between attention and multisensory processing in children remains largely unexplored. It is noteworthy that, like multisensory processes attention also follows a very protracted developmental trajectories with related neural mechanisms continuing to mature throughout late childhood and adolescence (e.g., Abundis-Gutierrez, Checa, Castellanos, \& Rosario Rueda, 2014; Cromer, Schembri, Harel, \& Maruff, 2015; Gogtay et al., 2004; Lebel \& Beaulieu, 2011; Manly et al., 2001; Nagy, Westerberg, \& Klingberg, 2004). Child developmental studies have also suggested that the maturation of multisensory processing and their interplay with learning and other cognitive abilities may partly depend on children's attention abilities (Barutchu et al., 2011; Broadbent, White, Mareschal, \& Kirkham, 2017), but these suggestions have been largely speculative rather than experimentally based. It is therefore important to develop an understanding of the relationship between attention, multisensory processing, and children's intellectual abilities, as this will inform us about factors that play an important role in the development of children's perceptual and cognitive abilities, and potentially lead to better learning and rehabilitation strategies.

This study aimed to explore the roles of auditory sustained and visual selective attention in the development of different multisensory processes given that the 
trajectory of maturation of multisensory processes have been shown to depend on whether multisensory tasks are verbal or non-verbal in nature (Tremblay et al., 2007). In particular, we aimed to investigate the developmental relationships between attention and multisensory processes when comparing the verbal-speech-based McGurk Effect and the non-verbal SB Effect. We also investigated the effect of temporal asynchronies between the auditory and visual information on the SB Effect. Attention was defined in terms of three subscales of the standardized Test of Everyday Attention for Children (TEA-Ch ) that is designed to assess attention in school-age children. The TEA-Ch examines aspects of static selective visual spatial attention, sustained auditory attention, and dual auditory and visual attention abilities. We hypothesized that the McGurk and SB Effects would become more pronounced with age. Based on findings in adults, we also predicted that children with better visual and dual audiovisual attention abilities would be more likely to perceive the McGurk Effect (Andersen et al., 2009; Fairhall \& Macaluso, 2009). For the SB Effect, we predicted that reports of bouncing objects would be higher when the sound is presented in synchrony with the objects touching and that the perception of bouncing objects would be higher in children with better auditory attention abilities. The relationships between attention, multisensory processing, and children's general intellectual abilities were also assessed.

\section{Method}

\section{Participants}

In total, 66 children aged 7-13 were recruited from local independent (nongovernment) schools in Victoria, Australia. All participants were right-handed with 
no reported neurological or psychological disorders. All participants spoke English as a first language and were assessed on the Wechsler Intelligence Scale for Children (WISC-IV; Wechsler, 2003). All but three participants, who were excluded from the study, had normal hearing and normal or corrected to normal vision. A further 10 children were excluded from the data analysis due to low performances on IQ subscales (see below for details).

Informed consent was obtained from the parents of children who participated. All procedures were carried out according to prescribed ethical standards and the protocols were approved by the Human Research and Ethics Committees of La Trobe University and The Royal Victorian Eye and Ear Hospital. Approval to conduct this research was also obtained from the Catholic Education Office, Melbourne, Australia.

\section{Screening and psychometric measures}

Screening measures were carried out on all participants to ensure that they could detect tones at a minimum of $20 \mathrm{~dB}$ sound pressure level at each octave step between frequencies $250 \mathrm{~Hz}$ to $8000 \mathrm{~Hz}$ using a calibrated audiometer with sound attenuated headphones. Visual screening measures included the assessment of near and distance acuity, binocular vision, and color vision. Children with high contrast visual acuity less than 6/7.5 were excluded from the study.

The pro-rated version of the WISC-IV (Wechsler, 2003) was used to measure children’s Full Scale Inteligence Quotient (FSIQ) and indices on the four subscales: Verbal Comprehension Index (VCI), Perceptual Reasoning Index (PRI), Working Memory Index (WMI), and Processing Speed Index (PSI). Due to the timed and forced-choice nature of the SB task, only high functioning children who scored above 80 on all IQ indices were included in the final analysis. Note that an IQ indices 
between 70-79 are classified as 'very low' functioning and considered as an indication of a borderline disability (Wechsler, 2003). In total 10 children failed this criterion: 3 children on the WMI index, 3 children on the PSI index, 1 child on the VCI index, 1 child on both the WMI and PRI index, 1 child on both FSIQ and the PSI index, and 1 child on both the PSI and PRI index. See Table 1 for the details of the 53 participants included in the final analyses.

Three subtests of The Test of Everyday Attention for Children (TEA-Ch) were used to assess auditory and visual attention abilities: Sky Search (SS), Score, and Sky Search Dual Task (SSDT) (Manly et al., 2001; Manly, Robertson, Anderson, \& Nimmo-Smith, 1998). The TEA-Ch and these indices were chosen because they provided age-standardized scores of visual, auditory, and divided audiovisual attention. The SS is a timed test of visual selective attention, where participants are presented with an A3 sheet with numerous pairs of symbols of spacecraft. Children were asked to search and circle as many pairs of matching spacecraft symbols among unmatched distractor pairs as quickly as possible. The timer was stopped when children indicated that they had completed the test by marking a box in the bottom left corner of the A3 SS sheet. Participants also completed a second version without unmatched distractors to control for motor speed. The SS index is calculated by subtracting the time taken per item on the control version from the attentionally demanding version with distractors. The Score subtest assesses sustained auditory attention, requiring participants to silently count a sequence of randomly presented sounds ranging from 9 to 15 across 10 trials. The score is derived from the number of trials with correct responses. The SSDT task is a dual auditory and visual attention task in which participants perform the SS and the Score subtests simultaneously. The score depended on the time taken to complete the trial, the number of tones completed 
and correctly reported, and the number of correctly circles matching spacecraft symbols. Three age-standardized indices, ranging between 1 and 19, were derived for each subtest and used for further analyses.

\section{The McGurk Effect task}

The McGurk Effect stimuli consisted of video recordings of a female articulating /ba-ba/, /da-da/, and /ga-ga/. Videos were edited to show the actor at the central point of fixation on a 15-inch laptop screen (see Figure 1A for an example of the experimental set-up). The classic McGurk Effect was created by dubbing in the sound for /ba-ba/ in the video with the articulation for /ga-ga/. The predicted auditory perceptual illusion was /da-da/. In total, four videos were used in the task (i.e., three matching videos and one audiovisual incongruent video with /ba-ba/ sounds and /gaga/ lip movements). Initially, participants were randomly presented with the three matching videos as practice trials to ensure that all children could perceive and accurately report the three syllable sounds. This was followed by 15 trials of the incongruent video. Following the video presentations, participants were given five multiple-choice options to choose from: /ba-ba/ (response consistent with audition), /ga-ga/ (response consistent with vision), /da-da/ (the McGurk Effect), as well as /baga/ and /gaba/ (two responses consisting of the combination of the visual and auditory sounds). At the end of each trial, children were asked to verbalize what they heard and to choose one of the five options (i.e., we used the phrases 'What did you hear?' and/or 'What did the lady say?' to ensure that the children understood the instructions). The responses were entered either by the child or the experimenter (if the child was too slow or unable to use the mouse) by clicking on the matching response. The participant or the experimenter initiated successive trials by clicking the 'NEXT' button on the screen following a response (see Figure 1A). Three 
children’s McGurk data were lost due to computer failure. Therefore, 52 children were included in the McGurk analysis.

A

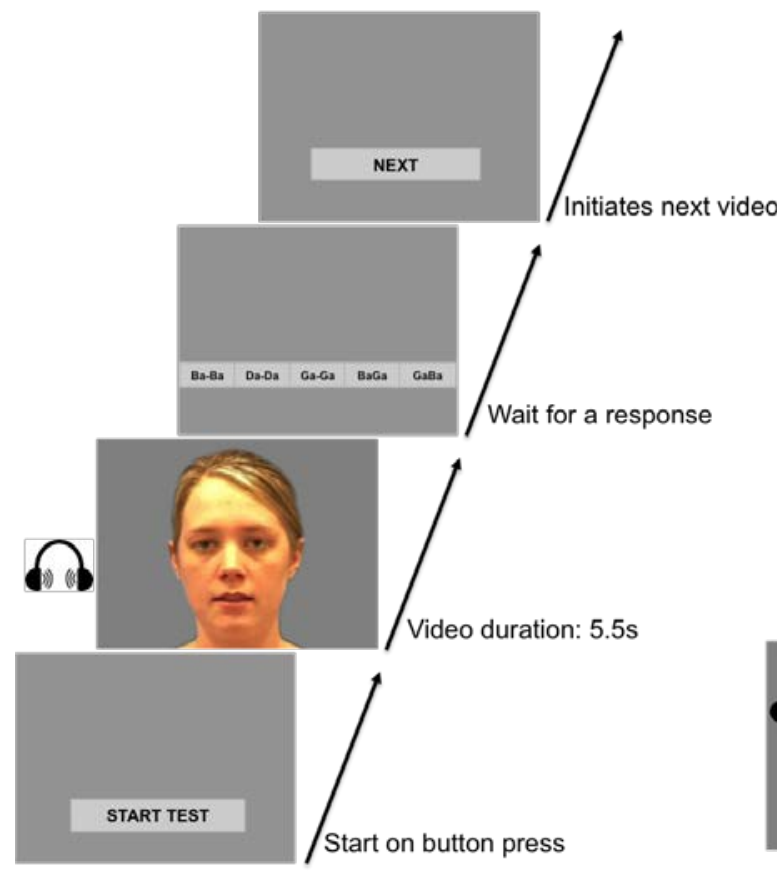

B

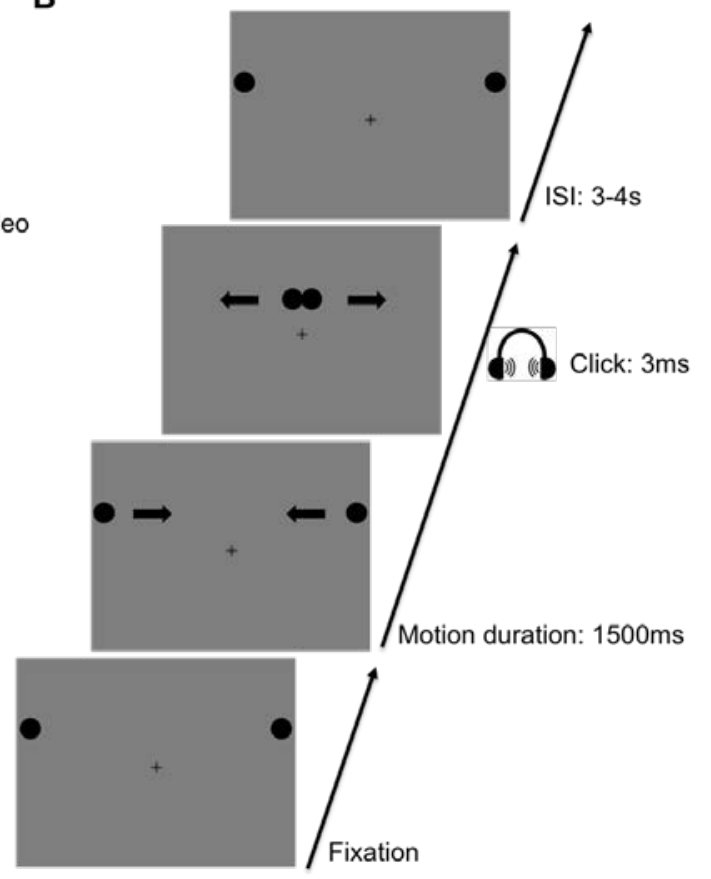

Figure 1. A. An illustration of the experimental trial setup for the McGurk. Note that the video initiated and finished with the actor centrally fixating for approximately 2 seconds and the speech duration was approximately 1.5 seconds in the middle of the video. B. An illustration of the Stream-Bounce (SB) tasks. Note that the arrows demonstrate the chronological sequence but were not presented as part of the task. The 3 ms click was presented 300 or 600 ms before or after the collision, or at the point of collision. 
The Stream-Bounce (SB) Effect task consisted of two laterally positioned solid black discs measuring $2^{\circ}$ of visual angle at a distance of $70 \mathrm{~cm}$ from the computer moniter with a grey background and a black centrally-positioned fixation cross measuring $0.5^{\circ}$ of visual angle. Visual stimuli were presented on a 21 -inch CRT monitor (see Figure 1B for an example of screen setup) at a refresh rate of $160 \mathrm{~Hz}$. The starting positions of the circles were $2^{\circ}$ above and $\pm 8^{\circ}$ lateral of the fixation cross. The two discs moved toward each other at a speed of $\sim 18^{\circ}$ per second. The auditory stimulus was a $3 \mathrm{~ms}$ tone pip $(1500 \mathrm{~Hz}$ at $70 \mathrm{~dB})$. In total, there were six types of trials: a visual-only trial and five audiovisual trials where the tone was presented either when the two discs touched/collided (0 ms asynchrony) or with asynchronies of \pm 300 or \pm 600 ms (note that negative values refer to the sound being presented before the point of collision, while positive values refer to after collision). Participants were randomly presented 15 trials of each of the six stimuli types (i.e., one visual only trial and 5 audiovisual trials with asynchronies) in one block of 90 stimuli. The inter-stimulus interval (ISI) between trials randomly varied between 3-4 sec. Participants were asked to make a forced-choice response using a button press with their right hand index and middle fingers to indicate whether they perceived the two discs to be passing (or streaming) through each other or bouncing off (colliding or hitting) each other, respectively. All participants were given at least 10 practice trials before initiating the final test block. Unfortunately, the SB data files of 7 children were lost due to computer failure. Therefore, the final SB data analysis consisted of 47 participants.

\section{Procedure}


Children were assessed individually in a quiet classroom during normal school hours in two 45-minute sessions, totaling approximately 1.5 hours of testing per child. All children initially had their audition and vision screened. The WISC-IV, TEA-Ch subscales, McGurk Effect task, and SB Effect task were administered in random order. For the computer tasks, children were seated $70 \mathrm{~cm}$ from the computer screen and asked to wear sound attenuating headphones. The experimenter was seated to the side and behind the participant, monitoring the participant to ensure attention to the screen at all times.

\section{Analyses}

For the McGurk-task, there were four possible response types with the five different responses: the McGurk Effect (the perception of /da/-/da/), responses consistent with audition (perception of /ba/-/ba/), responses consistent with vision (perception of /ga/-/ga/), and combination responses (perception of /baga/ or /gaba/). The percentages of audition, vision, and combination responses were very low with a median score across participants of $0 \%$ (indicating that these responses were never selected by more than half of the children), thus leading to violation of the assumption of normality. Therefore, overall differences in response types were assessed using the nonparametric Friedman test, followed by the Wilcoxon Sign Rank Test for pairwise comparisons. The relationships between the percentage of responses consistent with the McGurk Effect, age, attention indices (SS, Score and SSDT), and IQ indices (FSIQ, VCI, PRI, WMI and PSI) were assessed using correlations. In addition, backward regression was used to assess the optimal model that could predict the McGurk Effect with age, the three attention measures (SS, Score, and SSDT), and FSIQ as predictors. We chose backward regression because of the exploratory nature of the study (i.e., given that this was the first study of its kind, we were unable to use 
prior research to be more selective with our variables of interest). Backward regression identifies the optimal predictive model by initially inputting all variables into the model and then removing the least contributing variables one at a time until the optimal model is identified, while the alpha level is maintained at .05 (see Gravetter \& Wallnau, 2017; Tabachnick \& Fidell, 2007).

For the SB Effect, participants responded as 'bounce' or 'stream,' or participants made an 'error' response by pressing both buttons or failing to press a button. Error responses were very low and the correlation between the percentage of perceived stream and bounce responses was very high $(r=-0.97, p<.001)$, so only the percentages of bounce responses were included in further data analyses. Initially, a 2(bounce vs. error responses) x 6(no sound, -600ms, -300ms, 0ms, 300ms, 600ms) repeated measures ANOVA was used to assess the effect of auditory-visual synchrony on the SB Effect and the error in responding. Correlations were used to assess the relationships between the percentage of bounce responses at $0 \mathrm{~ms}$ asynchrony, age, attention indices (SS, Score, and SSDT), and IQ indices (FSIQ, VCI, PRI, WMI, and PSI). In addition, backward regression was used to assess the optimal model that could predict the SB Effect with age, the three attention measures (SS, Score, and SSDT), and FSIQ as predictors.

Multiple comparisons were corrected using the Benjamini-Hochberg criterion. 


\section{Results}

Table 1

Mean (SD), Minimum, Maximum and Range for age, IQ, and attention indices, and the Number of Females and Males(F/M).

\begin{tabular}{rccccc}
\hline \multicolumn{1}{c}{} & & Mean (SD) & Minimum & Maximum & Range \\
\hline \multirow{4}{*}{ IQ } & Age & $9.18(1.61)$ & 7.00 & 13.67 & 6.67 \\
& F/M & $30 / 23$ & & & \\
& & & & & \\
& FSIQ & $112.30(12.68)$ & 88 & 146 & 58 \\
VCI & $109.96(13.65)$ & 89 & 149 & 60 \\
PRI & $112.68(12.99)$ & 86 & 153 & 67 \\
WMI & $105.06(11.16)$ & 86 & 141 & 55 \\
PSI & $108.55(12.52)$ & 88 & 138 & 50 \\
Attention & & & & \\
SS & $9.57(2.90)$ & 2 & 19 & 17 \\
Score & $10.91(3.08)$ & 4 & 15 & 11 \\
SSDT & $8.32(4.06)$ & 1 & 18 & 17 \\
\hline
\end{tabular}

The overall means and standard deviations (SDs) of age, IQ, and attention indices for the group of children are presented in Table 1 . Table 2 shows the relationship between the attention indices, multisensory processing, age, and IQ. As expected, the IQ subscales, designed to assess different aspects of intellectual abilities (WISC-IV; Wechsler, 2003), showed moderate correlations with each other with an exception for the WMI and PSI indices ( $r=.27, p=.03$ ), which did not reach the Benjamini-Hochberg corrections for multiple comparisons. Given that the FSIQ index is a composite of all the subscales, it is not surprising that all four IQ subscales highly correlated with the FSIQ index.

Although all attention measures were standardized for age, the SSDT measure still showed a significant correlation with age $(r=.37, p=.004)$. The only significant correlation between the attention indices was between the SS and the Score index ( $r=$ 
$.33, p=.008)$. The correlation between SS and SSDT $(r=.28, p=.02)$ approached significance but failed to reach the adjusted Benjamini-Hochberg corrections for multiple comparisons. The observed low correlations between the attention indices suggest that they are tapping into different types of attention processes. Interestingly, only, the SS index was significantly correlated with the IQ PSI index $(r=.40, p=$ $.001)$. The correlation between the SS index and FSIQ $(r=.27, p=.02)$, SSDT and PSI index ( $r=.23, p=.047)$, and the Score and VCI index $(r=.26, p=.03)$ were borderline, not reaching significance once adjusted for multiple comparisons. The observed low correlations between the attention and the IQ indices also suggest that the different attention and IQ indices are tapping into different cognitive and intellectual processes. 
Table 2

Correlation matrix showing r-values, and p-values for age (number of participants are shown in brackets), for multisensory illusions (McGurk Effect and the SB Effect), attention measures (SS, Score and SSDT), and the IQ indices (FSIQ, VCI, PRI, WMI and PSI).

\begin{tabular}{|c|c|c|c|c|c|c|c|c|c|c|c|}
\hline \multirow{2}{*}{\multicolumn{2}{|c|}{$\begin{array}{c}\text { Age } \\
1\end{array}$}} & \multicolumn{2}{|c|}{ MS } & \multicolumn{3}{|c|}{ Attention } & \multicolumn{5}{|c|}{ IQ indices } \\
\hline & & 2 & 3 & 4 & 5 & 6 & 7 & 8 & 9 & 10 & 11 \\
\hline \multirow[t]{2}{*}{ 1. Age } & -- & $.28^{\wedge}$ & .22 & .06 & .01 & $.36^{*}$ & -.15 & $\begin{array}{l}.09 \\
\end{array}$ & -.13 & -.07 & -.04 \\
\hline & & $\begin{array}{c}.02 \\
\text { (51) }\end{array}$ & $\begin{array}{c}.07 \\
(47)\end{array}$ & $\begin{array}{c}.33 \\
(53)\end{array}$ & $\begin{array}{c}.47 \\
\text { (53) }\end{array}$ & $\begin{array}{l}.004 \\
(53)\end{array}$ & $\begin{array}{l}.15 \\
\text { (53) }\end{array}$ & $\begin{array}{c}.27 \\
\text { (53) }\end{array}$ & $\begin{array}{c}.17 \\
\text { (53) }\end{array}$ & $\begin{array}{c}.32 \\
\text { (53) }\end{array}$ & $\begin{array}{c}.38 \\
\text { (53) }\end{array}$ \\
\hline \multicolumn{12}{|c|}{ Multisensory Illusions } \\
\hline \multirow[t]{3}{*}{ 2. McGu } & & -- & -.03 & .19 & .15 & $.34 *$ & -.07 & -.10 & -.09 & -.13 & .15 \\
\hline & & & .43 & .09 & .14 & .007 & .31 & .24 & .26 & .18 & .15 \\
\hline & & & (45) & (51) & (51) & (51) & (51) & (51) & (51) & (51) & (51) \\
\hline \multirow[t]{3}{*}{ 3. SB } & & & -- & .03 & $.35 *$ & .15 & .17 & .20 & -.06 & .15 & .16 \\
\hline & & & & .43 & .008 & .16 & .12 & .09 & .34 & .15 & .14 \\
\hline & & & & (47) & (47) & (47) & (47) & (47) & (47) & (47) & (47) \\
\hline \multicolumn{12}{|c|}{ Attention indices } \\
\hline \multirow[t]{3}{*}{ 4. SS } & & & & -- & $.33 *$ & $.28 \wedge$ & $.27 \wedge$ & .18 & .21 & .08 & $.40 *$ \\
\hline & & & & & .008 & .02 & .02 & .10 & .07 & .27 & .001 \\
\hline & & & & & (53) & (53) & (53) & (53) & (53) & (53) & (53) \\
\hline \multirow[t]{3}{*}{ 5. Score } & & & & & -- & .20 & .11 & $.26^{\wedge}$ & -.03 & .05 & .11 \\
\hline & & & & & & .08 & .22 & .03 & .41 & .35 & .21 \\
\hline & & & & & & (53) & (53) & (53) & (53) & (53) & (53) \\
\hline \multirow[t]{3}{*}{ 6. SSDT } & & & & & & -- & .17 & .02 & .08 & .07 & $.23^{\wedge}$ \\
\hline & & & & & & & .11 & .43 & .29 & .30 & .05 \\
\hline & & & & & & & (53) & (53) & (53) & (53) & (53) \\
\hline \multicolumn{12}{|l|}{ IQ indices } \\
\hline \multirow[t]{2}{*}{ 7. FSIQ } & & & & & & & -- & $.78 * *$ & $.81^{* *}$ & $.65^{* *}$ & $.72 * *$ \\
\hline & & & & & & & & $\begin{array}{c}<.001 \\
(53)\end{array}$ & $\begin{array}{c}<.001 \\
(53)\end{array}$ & $\begin{array}{c}<.001 \\
(53)\end{array}$ & $\begin{array}{c}<.001 \\
(53)\end{array}$ \\
\hline \multirow[t]{2}{*}{ 8. VCI } & & & & & & & & -- & $.52 * *$ & $.45^{* *}$ & $.40 * *$ \\
\hline & & & & & & & & & $\begin{array}{c}<.001 \\
(53)\end{array}$ & $\begin{array}{c}<.001 \\
(53)\end{array}$ & $\begin{array}{l}.002 \\
(53)\end{array}$ \\
\hline \multirow[t]{2}{*}{ 9. PRI } & & & & & & & & & -- & $.44 * *$ & $.57^{* *}$ \\
\hline & & & & & & & & & & $\begin{array}{c}<.001 \\
(53)\end{array}$ & $\begin{array}{c}<.001 \\
(53)\end{array}$ \\
\hline \multirow{2}{*}{\multicolumn{2}{|c|}{ 10. WMI }} & & & & & & & & & -- & $.27^{\wedge}$ \\
\hline & & & & & & & & & & & $\begin{array}{r}.03 \\
(53)\end{array}$ \\
\hline
\end{tabular}

Note: ${ }^{*} p<.01,{ }^{* *} p<.001, \wedge=.01<p<.05$ ( $p$-values above the significance criterion using the Benjamini-Hochberg correction for multiple comparisons). Note that only 45 participants completed both of the McGurk and the SB task without computer failure. 
The correlation between the two multisensory illusions did not approach significance ( $r=.03, p=.43$ ), suggesting that the McGurk Effect and the SB Effect tap into different multisensory processes (see Table 2). As expected, age correlated with the McGurk Effect ( $r=.28, p=.02$ ), however, this failed to reach significance with the Benjamini-Hochberg correction for multiple comparisons. The correlation between age and the SB effect was not significant, $(r=.22, p=.07)$. The McGurk Effect significantly correlated only with the audiovisual attention SSDT index $(r=$ $.38, p=.003)$. The SB Effect, on the other hand, significantly correlated only with the auditory attention Score index $(r=.35, p=008)$.

\section{The McGurk Effect}

For the McGurk Effect task, responses consistent with the McGurk Effect $(/ \mathrm{da}-\mathrm{da} /)$ were most common $(M=49.10$, Median $=53.33, S D=39.34)$. Responses consistent with the auditory signal (/ba-ba/) $(M=28.08$, Median $=0, S D=40.35)$, combination responses (/baga/ or $/ \mathrm{gaba} /)(\mathrm{M}=12.31$, Median $=0, \mathrm{SD}=24.21)$, and visual responses $(/ g a-g a /)(M=11.03$, Median $=0, S D=24.98)$ were rare with more than half of the children never selecting these responses (Figure 2). The nonparametric Friedman Test showed that the different response types significantly differed $\left(\chi^{2}(3)=33.02, p<.001\right)$. Follow-up pairwise comparisons using Wilcoxon Sign Rank Tests showed that participants were significantly more likely to perceive the McGurk Effect than the visual signal $(p<.001)$ and the combination of the auditory and visual signal $(p<.001)$. Responses consistent with audition were significantly high than vision ( $p=.03$ ). Differences between McGurk and Auditory 
responses $(p=.11)$, auditory and combination responses $(p=.05)$, and visual and combination responses $(p=.67)$ did not reach significance.

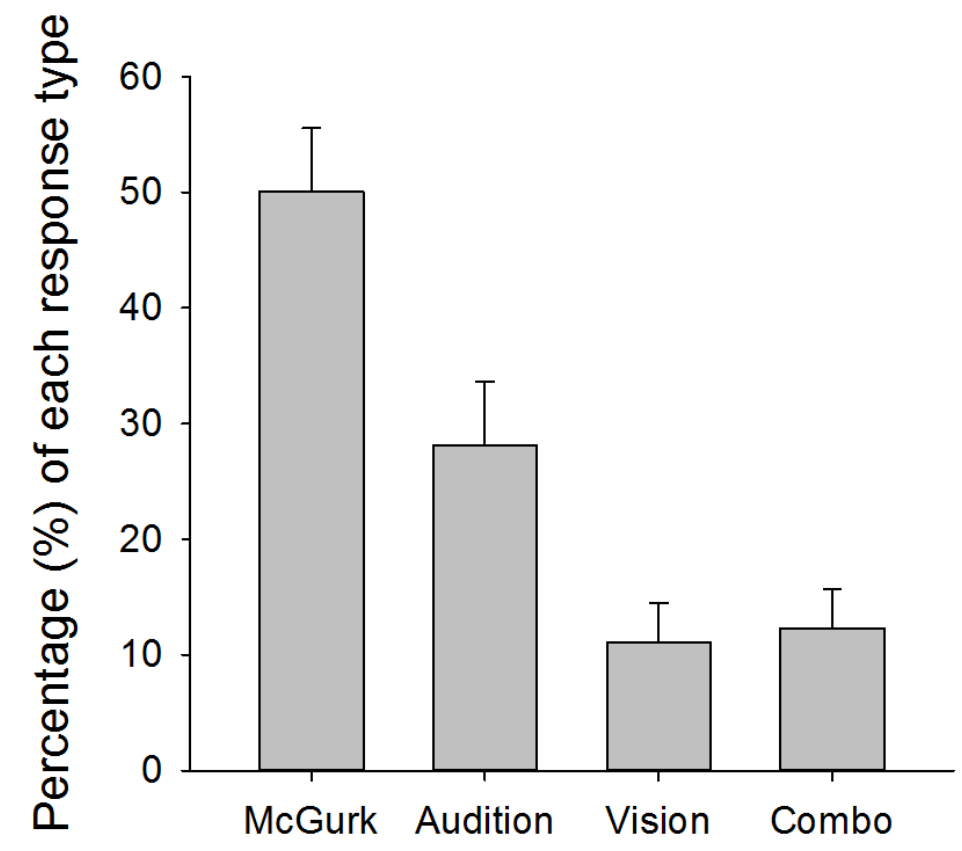

Figure 2. Percentage (+SEM) of responses consisted with the McGurk Effect, audition, vision and a combination response (combo).

As outlined above (see Table 2), the McGurk Effect significantly correlated with the attention SSDT index $(r=.34, p=.007)$, and it showed borderline significance with age with corrections for multiple comparisons $(r=.28, p=.02)$. A backward regression analysis identified three significant models once the two least contributing variables were removed: Score index (part $r=.08, p>.05$ ) and FSIQ index (part $r=.11, p>.05$ ). See Table 3. Although age (part $r=.19, p>.18$ ), the SS index (part $r=.10, p=.49$ ), and SSDT index (part $r=.23, p=.11$ ) were not significant predictors individually, collectively accounted for $16 \%$ of the McGurk 
Effect variance, $R=.40, F(3,48)=2.95, p=.04$. However, as can be seen from the reported part $r$ values shown in Table 3, the unique contributions of age, FSIQ, and the SS index were relatively small. The only significant predictor when all nonsignificant correlations were removed was the attention SSDT index accounting for $14.4 \%$ of the McGurk Effect variance, $R=.34, F(1,50)=6.54, p=.01$.

Table 3

R-values, F-statistics for the Backward Regression Model Outputs for the McGurk Effect and the SB Effect, and Part r-Values for the Variables Maintained in Each Model.

\begin{tabular}{|c|c|c|c|c|c|c|}
\hline \multirow[b]{2}{*}{ Models } & \multirow[b]{2}{*}{ R-value, F-statistic } & \multicolumn{5}{|c|}{ IV part $r, p$-value } \\
\hline & & Age & FSIQ & SS & Score & SSDT \\
\hline \multicolumn{7}{|c|}{ McGurk Effect } \\
\hline $1 \quad R$ & $\mathrm{R}=.42, F(5,46)=1.94, p=.11$ & $.15, p=.26$ & $-.11, p=.41$ & $.09, p=.50$ & $.08, p=.58$ & $.22, p=.10$ \\
\hline 2 & $\mathrm{R}=.42, F(4,47)=2.38, p=.07$ & $.15, p=.26$ & $-.11, p=.40$ & $.12, p=.38$ & -- & $.23, p=.09$ \\
\hline 3 & $R=.40, F(3,48)=2.95, p=.04$ & $.18, p=.18$ & -- & $.09, p=.49$ & -- & $.22, p=.11$ \\
\hline 4 & $\mathrm{R}=.38, F(2,49)=4.22, p=.02$ & $.18, p=.19$ & -- & -- & -- & $.26, p=.06$ \\
\hline 5 & $\mathrm{R}=.34, F(1,50)=6.54, p=.01$ & -- & -- & -- & -- & $.34, p=.01 *$ \\
\hline \multicolumn{7}{|c|}{ SD Effect } \\
\hline $1 \quad R$ & $\mathrm{R}=.49, F(5,41)=2.55, p=.04$ & $.27, p=.06$ & $.21, p=.13$ & $-.18, p=.18$ & $.37, p=.01 *$ & $.08, p=.96$ \\
\hline 2 & $\mathrm{R}=.49, F(4,42)=3.26, p=.02$ & $.28, p=.04 *$ & $.22, p=.11$ & $-.19, p=.18$ & $.37, p=.008^{*}$ & -- \\
\hline 3 & $\mathrm{R}=.45, F(3,43)=3.65, p=.02$ & $.26, p=.07$ & $.16, p=.24$ & -- & $.33, p=.02 *$ & -- \\
\hline 4 & $\mathrm{R}=.42, F(2,44)=4.70, p=.01$ & $.23, p=.1$ & -- & -- & $.36, p=.01 *$ & -- \\
\hline
\end{tabular}




\section{The Stream-Bounce Effect}

For the SB Effect task, error responses, where participants failed to respond or pressed both buttons, were very low across all sound conditions (Figure 3). Bounce responses were relatively much more reliable and significantly higher than error responses, $F(1,46)=80.27, p<.001, \eta^{2}=.64$. The main effect for sound asynchrony, $F(5,230)=18.81, p<.001, \eta^{2}=.29$, and the interaction effect between response type (error vs. bounce) and sound asynchrony were also significant, $F(5,230)=14.58, p<001, \eta^{2}=.24$. Follow-up simple effects analyses showed no significant differences in error responses across the different auditory asynchrony conditions. All sound conditions significantly increased bounce responses $(p<.001$ for all comparisons). In addition, pairwise comparisons showed that bounce responses were significantly higher for the 0 ms asynchrony condition than when the sound was presented $600 \mathrm{~ms}$ after the collision point (i.e., the $+600 \mathrm{~ms}$ asynchrony condition, $p=$ $.01)$. The difference between the $0 \mathrm{~ms}$ asynchrony and when the sound was presented 300 ms before the collision point (i.e., -300 ms asynchrony) approached significance $(p=.06)$. All other pairwise comparisons for asynchronies failed to reach significance $(p>.1$ for all). 


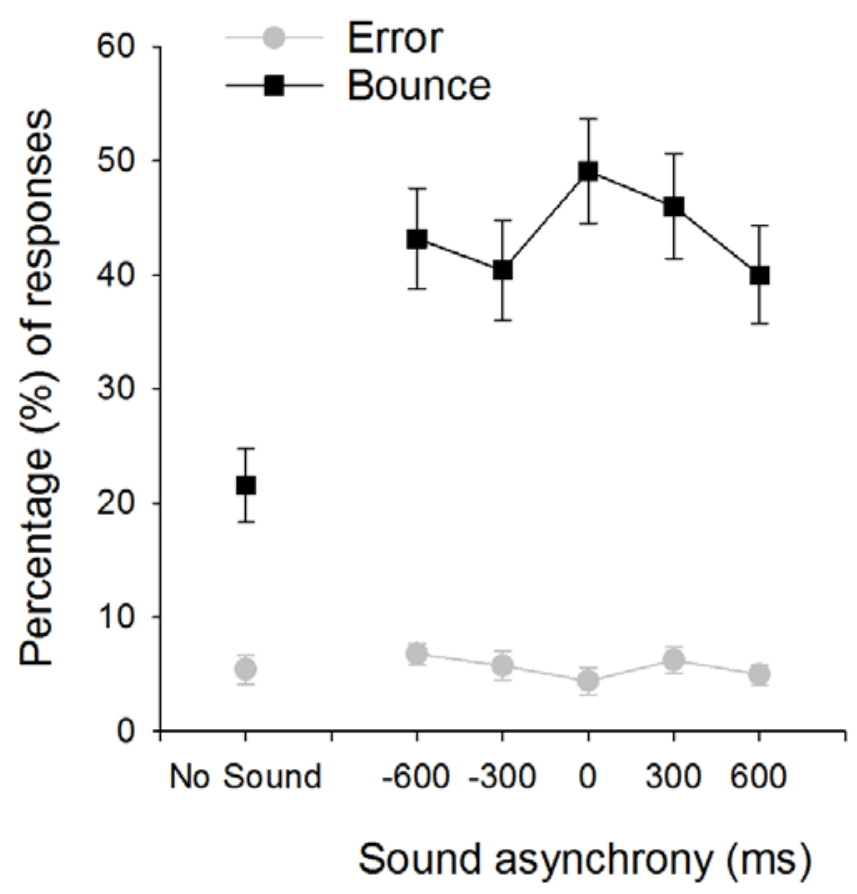

Figure 3. Mean percentage of error and bounce responses ( \pm SEM) without and with the sound.

The SB Effect showed a significant correlation with the sustained auditory attention Score index $(r=.35, p=.008)$, but the correlation between the SB Effect and age was not significant $(r=.22, p=.07$ ) (see Table 2). A linear backwards regression revealed four significant models (see Table 3). Collectively, age, SS, Score, SSDT, and FSIQ significantly predict the SB Effect, $R=.49, F(5,46)=2.55, p$ $=.04$. Analysis of part correlations identified the attention Score index as a significant predictor explaining $14.4 \%$ of variance (part $r=.37, p=.01$ ), and age approached significance explaining 7.3\% of the SB Effect variance (part $r=.27, p=.06$ ). The part correlations for FSIQ (part $r=.21, p>.05$ ), the SS index (part $r=-.18, p>.05$ ) and the SSDT index (part $r=.01, p>.05$ ) were not significance. The optimal significant model identified by backward regression included the attention Score 
index (part $r=.36, p=.01$ ) and age (part $r=.25, p=.1$ ), together accounting for $17.4 \%$ of the SB Effect variance, $R=.42, F(2,46)=4.70, p=.01$.

\section{Discussion}

This study is the first to show that multisensory processing is significantly related to children's attention abilities. This relationship is not only dependent on the sensory bias of the multisensory task but also on attention type. While the McGurk Effect is designed for visual perturbation of auditory information, our results indicate that performance is related to dual audiovisual attention abilities. The Stream-Bounce (SB) Effect is a visual task that is perturbed by auditory stimuli and it is related to children's auditory sustained attention. Age and general intellectual abilities alone could not explain the observed relationships between attention and multisensory processing.

Consistent with prior research, the McGurk Effect appears increase with age (McGurk \& MacDonald, 1976; Tremblay et al., 2007); however, this correlation between age and the McGurk Effect failed to reach significance following BenjaminiHochberg corrections for multiple comparisons, indicating the likelihood of a Type II error. We also found the McGurk effect to be significantly correlated with children's dual audiovisual attention abilities. The correlation between the McGurk Effect and selective spatial attention (i.e., the SS index) also approached significance, unlike the correlation with sustained auditory attention (the Score index). This finding is consistent with prior research that suggests attention has a binding role in the audiovisual integration of speech information (Alsius et al., 2005). It is also consistent with studies that have shown visual spatial attention and audiovisual temporal 
synchrony to jointly interact and influence McGurk perceptions (Andersen et al., 2009; Buchan \& Munhall, 2011; Fairhall \& Macaluso, 2009). The McGurk Effect is an illusory multisensory perception, whereby the perceived sound (phoneme) is altered by visual cues (lip movements). Therefore, the better that children can attend and process visual-spatial speech cues and integrate this information with audition, the more likely they are to perceive the McGurk Effect illusion. The current results suggest that children who are able to simultaneously attend to information in visual and auditory modalities in the broader sense can also experience enhanced integration of speech information and hence have a greater tendency to perceive the McGurk illusion.

The SB Effect illusion did not significantly correlate with age, and so showing a weaker relationship with age than the McGurk Effect. Also, although age pre se did not significantly contribute to the SB Effect on its own, the backward regression model maintained age as a predictor in the final model, thus, suggesting that age contributes to the prediction of the SB Effect. This suggests that different aspects of multisensory effects continue to develop throughout childhood, even though the McGurk and the SB Effect did not correlate with each other. This lack of correlation between the two multisensory illusions and the differences in their correlations with age suggests that the two illusions are tapping into different multisensory processes. This is also likely to reflect a dissociation in the developmental trajectories for verbal and non-verbal multisensory processes (Tremblay et al., 2007). The SB Effect emerges in infancy (Scheier, Lewkowicz, \& Shimojo, 2003) and it may be dependent on fundamentally simpler auditory and visual dynamics than the McGurk Effect. It is of an evolutionary advantage to detect and quickly react to colliding object from a very young age (e.g., when learning to grasp objects and to walk). Thus, the SB Effect 
may be driven from the older subcortical multisensory systems, such as the superior colliculus, that plays an important role in motor coordination, and which develops relatively early postnatally (e.g., Stein \& Stanford, 2008; Wallace, Perrault, Hairston, \& Stein, 2004). Indeed cortical brain regions involved in motor and motion perception also develop relatively early compared to temporal and associations brain areas that well known to be involved in language and speech (e.g., Gogtay et al., 2004). Further research is needed to better establish any differences in the developmental trajectory between the McGurk and the SB Effect.

The SB Effect was significantly related to children's auditory sustained attention abilities, but interestingly not to their visual-spatial and audiovisual attention abilities. The regression model identified only age and the Score index as significant contributors to the prediction of the SB Effect in children. This finding is consistent with the assumption that the baseline perception of streaming objects is dependent on visual attention, and that the perception of bouncing objects is increased by auditory attention capturing or distracting attention from the visual sense (Shimojo et al., 2001). In the SB Effect, visual perception is altered by sound. Therefore, for children to perceive the SB illusion, multisensory processes may be more strongly dependent on the stability of auditory sustained attention to capture attention and integrate the brief sound with the moving objects and, thus, perceive the objects as bouncing.

We also noted a very wider temporal window of audiovisual integration for the SB Effect than previously reported in adults (Fujisaki, Shimojo, Kashino, \& Nishida, 2004; Grove, Kawachi, \& Kenzo, 2012; Sekuler et al., 1997). In adults, multisensory processes can also have very wide temporal integration windows of up to $800 \mathrm{~ms}$ to allow for the merging of information over long distances, such as thunder and lightning, or objects that may be colliding in far distances (M. T. Wallace 
et al., 2004). In this study, children’s perception of 'bouncing objects’ was significantly greater than that of streaming objects even with asynchronies of up to $600 \mathrm{~ms}$, suggesting that children are overgeneralizing the illusion over a very wide time window. This finding is consistent with other studies that have shown wider time windows of integration in children than adults (Chen et al., 2016; Hillock, Powers, \& Wallace, 2011; Hillock-Dunn \& Wallace, 2012). Like Chen et al (2016), we also observed a trend of a wider window of integration when the visual signal preceded audition. Although this trend did not reach significance, it is important to note the possibility of a Type II error as the observed trend is consistent, not only with prior research, but also with the ecologically valid expectation of audiovisual signals reaching the vision (e.g., first lip-move or objects collide) before audition (e.g., causing the production of speech or the sound 'bang', respectively) (van Eijk, Kohlrausch, Juola, \& van de Par, 2008). Thus, one would naturally expect greater tolerance for the delay of auditory signals in multisensory integration. Alternatively, this finding may be consistent with Innes-Brown et al. (2011), who showed an increase in the probability of non-verbal flash-beep illusions and, thus, reduced multisensory selectivity in children. Given that children are slower and less accurate, not only at processing auditory and visual information (Barutchu et al., 2010) but also at shifting their attention, the temporal window of integration in children may remain relatively flexible as children develop. Future studies are needed to investigate differences in the temporal window of the SB Effect in adults and children to determine whether children temporally integrate information less selectively with larger time windows of integration.

In this study, we used the TEA-Ch to assess attention, which is a pen and paper test often used clinically to assist in the diagnosis of disorders related to 
attention. Nevertheless, we still observed significant relationships between children's attention and our psychophysical assessment of multisensory processing. We also used the WISC-IV to assess and control for children's intellectual abilities, and as expected and consistent with the test manual, we observed moderate to high correlations between the IQ indices. However, only the selective visual attention index (SS) and the SSDT index showed relatively lower correlations with the FSIQ index and processing speed index (PSI), most likely related to the timed nature of all three indices (i.e., children were asked to comple all tasks as fast and accurately as possible while being timed on their perfromance). The low correlations between the attention and IQ indices are not surprising given that in this study we only considered low to high functioning children with IQ indices above 80 on all subscales. Also, the TEA-Ch attention indices are designed to assess attention with minimal load on verbal, working memory and perceptual reasoning abilities, thus, further explaining the observed low correlations between attention and IQ indices. The multisensory illusions also showed low non-significant correlations with children’s intellectual abilities. This finding is consistent with the Barutchu et al. (2009) study that showed a non-significant relationship with children's multisensory motor enhancements and non-verbal intellectual abilities, though a follow-up study has shown that general intellectual abilities may be related to children’s ability to integrate audiovisual information, but only when auditory noise is considered (Barutchu et al., 2011) and when the tasks involve higher cognitive processes, such as associative learning (Barutchu, Fifer, Shivdasani, Crewther, \& Paolini, in press). These findings suggest that multisensory signals that give rise to multisensory illusions, such as the McGurk Effect and SB Effect merge early during signal processing and are not influenced by higher cognitive processes in high functioning children. Since perceptual, cognitive, 
behavioral and neural processes related to multisensory integration and attention are multifaceted and dependent on the task and the signal type (e.g., Calvert, 2001; Driver \& Noesselt, 2008; Talsma et al., 2010), the relationships between attention, multisensory integration and IQ are also likely to differ across tasks. For example, the relationship between attention, IQ and multisensory processing is likely to differ when multisensory tasks with greater cognitive load are employed (e.g., multisensory associative learning tasks). Further research is needed to assess the generalizability of these findings to other multisensory and attention processes, and to children with very low intellectual abilities.

In this study, we were limited by the TEA-Ch in our ability to assess spatial and sustained attention within each sensory system. Future studies could use alternative attention measures to assess sustained and spatial attention within each sensory system to dissociate whether it is the stability of attention over time and space, or if it is the ability to focus attention within a particular sensory system that contributes to the multisensory processes giving rise to the McGurk and SB Effects. We were also limited in our ability to assess multisensory illusions in very low functioning children with IQ below 80 due to the fact that we employed a timed forced choice paradigm for the SB Effect. In this task, the imposed time limit to respond (i.e, 3-4 s) meant that children with very low IQs and processing speed abilities may not have been able to decide and respond in time. Indeed when the analysis is run with these very low IQ children included only the SB Effect analysis is affected with the processing speed index (PSI) approaching significance and the relationship between the SB Effect and the attention Score measure weakened $(p=$ .05 for both, analysis not reported here). In contrast, the McGurk Effect is not affected because children could take as long as they needed to respond. This suggests that 
timed forced-choice paradigms can mask relationships between multisensory illusions and other processes even in late childhood, particularly, in very low IQ children who may employ alternative strategies, like guessing, to respond faster. Future studies should investigate the relationship between IQ, attention and multisensory illusions without imposing time restrictions on responses as these relationships may differ in children with very low intellectual abilities.

In conclusion, the relationship between multisensory processing and children's attention abilities was dependent not only on the multisensory task, but also on attention type. The McGurk Effect, where visual information alters auditory perception, was related to audiovisual attention abilities. On the other hand, when visual perception was altered by sound, as in the Stream-Bounce Effect, multisensory processes were related to auditory sustained attention abilities. Further research is needed to dissociate whether the relationship between attention and multisensory processing is specific to the sensory system or whether sustained, spatial, and dual attention processes in general can account for multisensory integration, not only in high functioning children, but children with perceptual and cognitive deficits. This knowledge is important as it has the potential to facilitate the development of better learning environments and rehabilitation strategies for children. 


\section{References}

Abundis-Gutierrez, A., Checa, P., Castellanos, C., \& Rosario Rueda, M. (2014). Electrophysiological correlates of attention networks in childhood and early adulthood. Neuropsychologia, 57, 78-92. doi:

10.1016/j.neuropsychologia.2014.02.013

Alsius, A., Navarra, J., Campbell, R., \& Soto-Faraco, S. (2005). Audiovisual integration of speech falters under high attention demands. Curr Biol, 15(9), 839-843. doi: 10.1016/j.cub.2005.03.046

Andersen, T. S. , Tiippana, K. , Laarni, J. , Kojo, I. , \& Sams, M. . (2009). The role of visual spatial attention in audiovisual speech perception. Speech Communication, 51(2), 184-193.

Bahrick, L. E., \& Lickliter, R. (2000). Intersensory redundancy guides attentional selectivity and perceptual learning in infancy. Dev Psychol, 36(2), 190-201.

Barutchu, A., Crewther, D. P., \& Crewther, S. G. (2009). The race that precedes coactivation: development of multisensory facilitation in children. Dev Sci, 12(3), 464-473. doi: 10.1111/j.1467-7687.2008.00782.x

Barutchu, A., Crewther, S. G., Fifer, J., Shivdasani, M. N., Innes-Brown, H., Toohey, S., .. Paolini, A. G. (2011). The relationship between multisensory integration and IQ in children. Dev Psychol, 47(3), 877-885. doi: 10.1037/a0021903

Barutchu, A., Danaher, J., Crewther, S. G., Innes-Brown, H., Shivdasani, M. N., \& Paolini, A. G. (2010). Audiovisual integration in noise by children and adults. J Exp Child Psychol, 105(1-2), 38-50. doi: 10.1016/j.jecp.2009.08.005 
Barutchu, A., Fifer, J. M., Shivdasani, M. N., Crewther, S. G., \& Paolini, A. G. (in press). The interplay between multisensory associative learning and IQ in children. Child Dev.

Brandwein, A. B., Foxe, J. J., Russo, N. N., Altschuler, T. S., Gomes, H., \& Molholm, S. (2011). The development of audiovisual multisensory integration across childhood and early adolescence: a high-density electrical mapping study. Cereb Cortex, 21(5), 1042-1055. doi: 10.1093/cercor/bhq170

Bremner, A. J., Lewkowicz, D. J., \& Spence, C. (2012). Multisensory development. Oxford: Oxford University Press.

Broadbent, H. J., White, H., Mareschal, D., \& Kirkham, N. Z. (2017). Incidental learning in a multisensory environment across childhood. Dev Sci. doi: 10.1111/desc. 12554

Buchan, J. N., \& Munhall, K. G. (2011). The influence of selective attention to auditory and visual speech on the integration of audiovisual speech information. Perception, 40(10), 1164-1182. doi: 10.1068/p6939

Calvert, G. A. (2001). Crossmodal processing in the human brain: insights from functional neuroimaging studies. Cereb Cortex, 11(12), 1110-1123.

Chen, Y. C., Shore, D. I., Lewis, T. L., \& Maurer, D. (2016). The development of the perception of audiovisual simultaneity. J Exp Child Psychol, 146, 17-33. doi: 10.1016/j.jecp.2016.01.010

Cromer, J. A., Schembri, A. J., Harel, B. T., \& Maruff, P. (2015). The nature and rate of cognitive maturation from late childhood to adulthood. Front Psychol, 6, 704. doi: 10.3389/fpsyg.2015.00704 
Downing, H. C., Barutchu, A., \& Crewther, S. G. (2014). Developmental trends in the facilitation of multisensory objects with distractors. Front Psychol, 5, 1559. doi: 10.3389/fpsyg.2014.01559

Driver, J., \& Noesselt, T. (2008). Multisensory interplay reveals crossmodal influences on 'sensory-specific' brain regions, neural responses, and judgments. Neuron, 57, 11-23.

Driver, J., \& Spence, C. (1998). Cross-modal links in spatial attention. Philos Trans $R$ Soc Lond B Biol Sci, 353(1373), 1319-1331. doi: 10.1098/rstb.1998.0286

Fairhall, S. L., \& Macaluso, E. (2009). Spatial attention can modulate audiovisual integration at multiple cortical and subcortical sites. Eur J Neurosci, 29(6), 1247-1257. doi: 10.1111/j.1460-9568.2009.06688.x

Foreman, N., \& Fielder, A. (1989). Intermodal enhancement of stimulus localisation in infants born prematurely. Percept Mot Skills, 69(1), 43-50. doi: 10.2466/pms.1989.69.1.43

Fujisaki, W., Shimojo, S., Kashino, M., \& Nishida, S. (2004). Recalibration of audiovisual simultaneity. Nat Neurosci, 7(7), 773-778. doi: 10.1038/nn1268

Gogtay, N., Giedd, J. N., Lusk, L., Hayashi, K. M., Greenstein, D., Vaituzis, A. C., . . . Thompson, P. M. (2004). Dynamic mapping of human cortical development during childhood through early adulthood. Proc Natl Acad Sci U S A, 101(21), 8174-8179. doi: 10.1073/pnas.0402680101

Goldberg, M. E., \& Wurtz, R. H. (1972). Activity of superior colliculus in behaving monkey. II. Effect of attention on neuronal responses. J Neurophysiol, 35(4), 560-574. doi: 10.1152/jn.1972.35.4.560

Gori, M., Del Viva, M., Sandini, G., \& Burr, D. C. (2008). Young children do not integrate visual and haptic form information. Current Biology, 6(18), 694-698. 
Gravetter, F. J., \& Wallnau, L. B. (2017). Statistics for the Behavioral Sciences (10th ed.). Boston, MA: Cengage Learning.

Grove, P. M., Kawachi, Y., \& Kenzo, S. (2012). The stream/bounce effect occurs for luminance- and disparity-defined motion targets. Perception, 41(4), 379-388. doi: $10.1068 / p 6808$

Hillock, A. R., Powers, A. R., \& Wallace, M. T. (2011). Binding of sights and sounds: age-related changes in multisensory temporal processing. Neuropsychologia, 49(3), 461-467. doi: 10.1016/j.neuropsychologia.2010.11.041

Hillock-Dunn, A., \& Wallace, M. T. (2012). Developmental changes in the multisensory temporal binding window persist into adolescence. Dev Sci, 15(5), 688-696. doi: 10.1111/j.1467-7687.2012.01171.x

Hillyard, S. A., Stormer, V. S., Feng, W., Martinez, A., \& McDonald, J. J. (2016). Cross-modal orienting of visual attention. Neuropsychologia, 83, 170-178. doi: 10.1016/j.neuropsychologia.2015.06.003

Innes-Brown, H., Barutchu, A., Shivdasani, M. N., Crewther, D. P., Grayden, D. B., \& Paolini, A. G. (2011). Susceptibility to the flash-beep illusion is increased in children compared to adults. Dev Sci, 14(5), 1089-1099. doi: 10.1111/j.1467-7687.2011.01059.x

James, W. . (1890). The Principles of Psychology. New York: Henry Holt.

Lebel, C., \& Beaulieu, C. (2011). Longitudinal development of human brain wiring continues from childhood into adulthood. J Neurosci, 31(30), 10937-10947. doi: 10.1523/JNEUROSCI.5302-10.2011

Manly, T., Anderson, V., Nimmo-Smith, I., Turner, A., Watson, P., \& Robertson, I. H. (2001). The differential assessment of children's attention: the Test of 
Everyday Attention for Children (TEA-Ch), normative sample and ADHD performance. J Child Psychol Psychiatry, 42(8), 1065-1081.

Manly, T., Robertson, I. H., Anderson, V., \& Nimmo-Smith, I. (1998). Test of Everyday Attention for Children (TEA-Ch): Pearson Education.

McGurk, H., \& MacDonald, J. (1976). Hearing lips and seeing voices. Nature, 264(5588), 746-748.

Muir, D., \& Field, J. (1979). Newborn infants orient to sounds. Child Dev, 50(2), 431436.

Nagy, Z., Westerberg, H., \& Klingberg, T. (2004). Maturation of white matter is associated with the development of cognitive functions during childhood. $J$ Cogn Neurosci, 16(7), 1227-1233. doi: 10.1162/0898929041920441

Nardini, M., Bales, J., \& Mareschal, D. (2016). Integration of audio-visual information for spatial decisions in children and adults. Dev Sci, 19(5), 803816. doi: 10.1111/desc.12327

Nardini, M., Bedford, R., \& Mareschal, D. (2010). Fusion of visual cues is not mandatory in children. Proc Natl Acad Sci U S A, 107(39), 17041-17046. doi: 10.1073/pnas.1001699107

Ross, L. A., Molholm, S., Blanco, D., Gomez-Ramirez, M., Saint-Amour, D., \& Foxe, J. J. (2011). The development of multisensory speech perception continues into the late childhood years. Eur J Neurosci, 33(12), 2329-2337. doi: 10.1111/j.1460-9568.2011.07685.x

Scheier, C., Lewkowicz, D. J., \& Shimojo, S. (2003). Sound induces perceptual reorganization of an ambiguous motion display in human infants. Dev Sci, 6(3), 233-244. 
Sekuler, R., Sekuler, A. B., \& Lau, R. (1997). Sound alters visual motion perception. Nature, 385(6614), 308. doi: 10.1038/385308a0

Shimojo, S., Watanabe, K., \& Scheier, C. (2001). The resolution of ambiguous motion: Attention modulation and development. In J. Braun, C. Koch \& J. L. Davis (Eds.), Visual attention and cortical circuits (pp. 243-264). Cambridge: The MIT Press.

Spence, C., \& Driver, J. (1996). Audiovisual links in endogenous covert spatial attention. J Exp Psychol Hum Percept Perform, 22(4), 1005-1030.

Stein, B. E., \& Stanford, T. R. (2008). Multisensory integration: current issues from the perspective of the single neuron. Nat Rev Neurosci, 9, 255-266.

Tabachnick, B. G., \& Fidell, L. S. . (2007). Using multivariate statistics. (5th ed.). Boston, MA: Allyn \& Bacon/Pearson Education.

Talsma, D. (2015). Predictive coding and multisensory integration: an attentional account of the multisensory mind. Front Integr Neurosci, 9, 19. doi: 10.3389/fnint.2015.00019

Talsma, D., Senkowski, D., Soto-Faraco, S., \& Woldorff, M. G. (2010). The multifaceted interplay between attention and multisensory integration. Trends Cogn Sci, 14(9), 400-410. doi: 10.1016/j.tics.2010.06.008

Tremblay, C., Champoux, F., Voss, P., Bacon, B. A., Lepore, F., \& Theoret, H. (2007). Speech and non-speech audio-visual illusions: a developmental study. PLoS One, 2(1), e742. doi: 10.1371/journal.pone.0000742

van Eijk, R. L., Kohlrausch, A., Juola, J. F., \& van de Par, S. (2008). Audiovisual synchrony and temporal order judgments: effects of experimental method and stimulus type. Percept Psychophys, 70(6), 955-968. 
Wallace, M. T., Roberson, G. E., Hairston, W. D., Stein, B. E., Vaughan, J. W., \& Schirillo, J. A. (2004). Unifying multisensory signals across time and space. Exp Brain Res, 158(2), 252-258. doi: 10.1007/s00221-004-1899-9

Wallace, M..T., Perrault, T. J., Hairston, W .D., \& Stein, B. E. (2004). Visual experience is necessary for the development of multisensory integration. $J$ Neurosci, 24(43), 9580-9584.

Wechsler, D. (2003). Wechsler Intelligence Scale for Children - Australian Standardisation (4th ed.). Sydney: Harcourt Assessment. 